01

\title{
Изотопические сдвиги уровней энергии в гелиеподобных многозарядных ионах
}

\author{
() Н.А. Зубова, М.Ю. Кайгородов, Ю.С. Кожедуб, А.В. Малышев, Р.В. Попов, И.М. Савельев, \\ И.И. Тупицын, В.М. Шабаев
}

Санкт-Петербургский государственный университет, 199034 Санкт-Петербург, Россия

e-mail: znataliam0@gmail.com

Поступила в редакцию 18.03.2020 г.

В окончательной редакции 18.03.2020 г.

Принята к публикации 25.03.2020 г.

\begin{abstract}
Прецизионно рассчитаны изотопические сдвиги уровней $n=1, n=2$ и соответствующих переходов в гелиеподобных многозарядных ионах. Полная величина изотопического сдвига определяется, главным образом, суммой полевого и массового сдвигов. Полевой сдвиг вычислен методом Дирака-Фока-Штурма с учетом взаимодействия конфигураций. Квантово-электродинамические поправки к этому вкладу учтены приближенно посредством использования соответствующих одноэлектронных формул. Массовый сдвиг вычислен как в рамках приближения Брейта, так и с учетом квантово-электродинамических вкладов, которые для тяжелых ионов становятся весьма значительными. В случае ионов тория и урана дополнительно учтены поправки на поляризацию и деформацию ядер.
\end{abstract}

Ключевые слова: изотопические сдвиги, релятивистская теория атома, зарядовые радиусы ядер.

DOI: $10.21883 /$ OS.2020.08.49702.115-20

\section{1. Введение}

Первые прецизионные измерения изотопических сдвигов в многозарядных ионах были выполнены в работах [1-3]. Наиболее высокая точность была достигнута в экспериментах по изотопическому сдвигу в бороподобном ионе аргона [4] и в литиеподобном ионе неодима [5]. Достигнутая точность позволила впервые протестировать релятивистские расчеты массового сдвига в многозарядных ионах и по порядку величины вплотную приблизилась к величине квантово-электродинамического (КЭД) вклада в эффект отдачи ядра. Исследование последнего вклада в тяжелых ионах представляет отдельный интерес, так как его расчет требует использования квантовой электродинамики в области сильной связи за рамками приближения, в котором ядро рассматривается как источник классического поля (картина Фарри), а не движущаяся частица. Кроме того, в работе [5] экспериментальные и теоретические значения изотопического сдвига в литиеподобном неодиме были использованы для определения разности зарядовых радиусов соответствующих изотопов. Ожидается, что экспериментальная точность измерений изотопических сдвигов в тяжелых ионах вплоть до урана $(Z=92)$ может быть улучшена примерно на порядок в рамках строящихся в настоящее время установок мегапроекта FAIR [6,7]. Все это безусловно свидетельствует об актуальности соответствующих теоретических расчетов.

В наших предыдущих работах [8-10] были выполнены прецизионные расчеты изотопических сдвигов уровней энергии литие-, боро- и бериллиеподобных многозаряд- ных ионов. Цель настоящей работы - расширить эти расчеты на случай гелиеподобных многозарядных ионов. Наиболее точные на сегодняшний день расчеты уровней энергии $n=1, n=2$ гелиеподобных многозарядных ионов были выполнены в работах [11-13]. Насколько нам известно, до настоящего времени прецизионных расчетов изотопических сдвигов в гелиеподобных многозарядных ионах не проводилось.

В работе используется релятивистская система единиц $(\hbar=c=1)$.

\section{2. Основные формулы}

Мы начнем рассмотрение с расчета полевого сдвига уровней энергии, который обусловлен эффектом конечности размера ядра. В стандартном релятивистском подходе расчет этого эффекта основывается на гамильтониане Дирака-Кулона-Брейта (ДКБ), который в случае двухэлектронного иона (атома) имеет вид

$$
H^{\mathrm{DCB}}=\Lambda^{(+)}\left[h_{1}^{\mathrm{D}}+h_{2}^{\mathrm{D}}+V_{12}\right] \Lambda^{(+)},
$$

где $\Lambda^{(+)}$- проектор на состояния, построенные из положительно-энергетических одночастичных решений уравнения Дирака с кулоновским или каким-либо эффективным потенциалом, который частично учитывает межэлектронное взаимодействие, $h^{\mathrm{D}}$ - одноэлектронный дираковский гамильтониан,

$$
\begin{aligned}
V_{12} & =V_{12}^{\mathrm{C}}+V_{12}^{\mathrm{B}} \\
& =\frac{\alpha}{r_{12}}-\alpha\left[\frac{\boldsymbol{\alpha}_{1} \cdot \boldsymbol{\alpha}_{2}}{r_{12}}+\frac{1}{2}\left(\boldsymbol{\alpha}_{1} \cdot \nabla_{1}\right)\left(\boldsymbol{\alpha}_{2} \cdot \nabla_{2}\right) r_{12}\right]
\end{aligned}
$$


- оператор межэлектронного взаимодействия в приближении Брейта, $\alpha$ - постоянная тонкой структуры.

Чтобы найти собственные значения энергии и волновые функции гамильтониана ДКБ, используем метод наложения конфигураций в базисе функций Дирака-ФокаШтурма [14,15]. Многоэлектронная волновая функция $\psi(\gamma J)(J-$ полный угловой момент, $\gamma-$ совокупность остальных квантовых чисел) раскладывается по большому набору базисных функций:

$$
\psi(\gamma J)=\sum_{\alpha} c_{\alpha} \Phi_{\alpha}(J)
$$

где $\Phi_{\alpha}(J)$ - собственные функции оператора квадрата полного углового момента $J^{2}$, отвечающие данной релятивистской конфигурации. Они получаются как линейные комбинации детерминантов Слейтера. Одноэлектронные орбитали $\left(\phi_{j}\right)$, отвечающие заполненным оболочкам, находятся из уравнений Дирака-Фока, в то время как виртуальные орбитали $\left(\tilde{\phi}_{j}\right)$ определяются из решений уравнений Дирака-Фока-Штурма:

$$
\left(h^{\mathrm{DF}}-\varepsilon_{j_{0}}\right) \tilde{\phi}_{j}=\lambda_{j} W(r) \tilde{\phi}_{j},
$$

где $h^{\mathrm{DF}}$ - оператор Дирака-Фока, $\varepsilon_{j_{0}}$ - одноэлектронная энергия занятой дирак-фоковской орбитали, $W(r)-$ постоянная весовая функция. Параметр $\lambda_{j}$ определяется как собственное значение оператора Штурма. Весовая функция $W(r)$ берется в виде

$$
W(r)=\frac{1-\exp \left[-(\mu r)^{2}\right]}{(\mu r)^{2}} .
$$

При таком выборе она регулярна в нуле и убывает как $1 / r^{2}$ при $r \rightarrow \infty$. При $\lambda_{j}=0$ штурмовская функция совпадает со ссылочной дирак-фоковской орбиталью $\left(\phi_{j_{0}}\right)$. Все штурмовские функции имеют одинаковую асимптотику при $r \rightarrow \infty$. В силу того, что оператор Штурма является эрмитовым и не содержит непрерывный спектр, штурмовские функции формируют полный дискретный базис одноэлектронных волновых функций.

Полевой сдвиг, который еще иногда называют эффектом объема, определяется разностью поправок на конечность размера ядра к атомным уровням двух различных изотопов. При расчетах этого сдвига распределение электрического заряда по ядру обычно моделируется сферически симметричным распределением Ферми:

$$
\rho(r, R)=\frac{N}{1+\exp [(r-c) / a]},
$$

где параметр $a$, как правило, полагают равным $a=2.3 /(4 \ln 3) \mathrm{fm}$, а параметры $N$ и $c$ определяются из заданного значения среднеквадратичного зарядового радиуса ядра $R=\left\langle r^{2}\right\rangle^{1 / 2}$ и условия нормировки: $\int d \mathbf{r} \rho(r, R)=1$. Создаваемый ядром потенциал определяется из решения уравнения Пуассона:

$$
V_{N}(r, R)=-4 \pi \alpha Z \int_{0}^{\infty} d r^{\prime} r^{\prime 2} \rho\left(r^{\prime}, R\right) \frac{1}{r_{>}},
$$

где $r_{>}=\max \left(r, r^{\prime}\right)$. Так как поправка на конечный размер ядра с погрешностью до $1 \%$ определяется величиной среднеквадратичного зарядового радиуса [16], разность энергий двух изотопов, находящихся в одном и том же электронном состоянии, удобно аппроксимировать формулой

$$
\delta E_{F S}=F \delta\left\langle r^{2}\right\rangle
$$

где $F$ - константа полевого сдвига и $\delta\left\langle r^{2}\right\rangle-$ разность средних квадратов зарядовых радиусов рассматриваемых изотопов. В соответствии с этим определением и теоремой вириала константа $F$ может быть также вычислена по формуле

$$
F=\left\langle\psi\left|\sum_{i} \frac{d V_{N}\left(r_{i}, R\right)}{d\left\langle r^{2}\right\rangle}\right| \psi\right\rangle
$$

где $\psi$ - волновая функция рассматриваемого состояния и суммирование идет по атомным электронам.

Как правило, использование формул (8), (9) является достаточным для достижения требуемой точности. Однако в случае большого различия радиусов ядер могут оказаться важными и разности более высоких моментов зарядового распределения $[17,18]$. В этом случае расчеты могут быть выполнены посредством прямого вычисления разности энергий связи для двух изотопов.

При вычислении полевого сдвига в тяжелых ионах следует также учитывать поправки на конечность размера ядра к квантово-электродинамическим вкладам, определяемым диаграммами собственной энергии и поляризации вакуума. В случае многозарядного иона это можно сделать приближенно посредством использования аналитических формул, полученных для водородоподобных ионов в [19]. Для изотопов урана могут быть также использованы аппроксимационые формулы, полученные в работе [20].

Массовый сдвиг атомных уровней обусловлен различными значениями вклада отдачи ядра (конечность массы ядра) в энергии связанных состояний. Так как, за исключением области малых значений заряда ядра $Z$, эффектами второго порядка по отношению массы электрона к массе ядра, $m / M$, можно пренебречь, то величину сдвига удобно представить в виде

$$
\delta E=K\left(\frac{1}{M_{1}}-\frac{1}{M_{2}}\right)=-\frac{\delta M}{M_{1} M_{2}} K
$$

где $K$ - так называемый $K$-фактор, $\delta M=M_{1}-M_{2}-$ разность масс ядер рассматриваемых изотопов. Полностью релятивисткие расчеты массового сдвига могут быть выполнены только в рамках квантовой электродинамики [21-28]. Такие расчеты предполагают использование теории возмущений по межэлектронному взаимодействию и являются довольно сложными [27,28]. Учет релятивистских поправок низшего порядка может быть выполнен в рамках подхода, основанного на гамильтониане ДКБ, посредством использования эффективного 


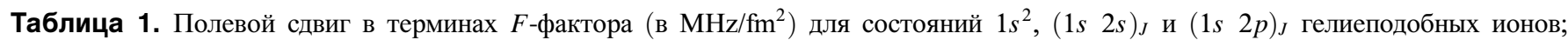
результаты получены методом наложения конфигураций в базисе функций Дирака-Фока-Штурма и не включают КЭД поправки

\begin{tabular}{c|c|c|c|c|c|c|c|c}
\hline Ион & $\left\langle r^{2}\right\rangle^{1 / 2}$ & $1 s^{2}$ & $(1 s 2 s)_{0}$ & $(1 s 2 s)_{1}$ & $\left(1 s 2 p_{1 / 2}\right)_{0}$ & $\left(1 s 2 p_{1 / 2}\right)_{1}$ & $\left(1 s 2 p_{3 / 2}\right)_{1}$ & $\left(1 s 2 p_{3 / 2}\right)_{2}$ \\
\hline $\mathrm{Mg}^{10+}$ & 3.0570 & $0.6481 \cdot 10^{5}$ & $0.3784 \cdot 10^{5}$ & $0.3802 \cdot 10^{5}$ & $0.3408 \cdot 10^{5}$ & $0.3408 \cdot 10^{5}$ & $0.3435 \cdot 10^{5}$ & $0.3408 \cdot 10^{5}$ \\
$\mathrm{Ca}^{18+}$ & 3.4776 & $0.5560 \cdot 10^{6}$ & $0.3204 \cdot 10^{6}$ & $0.3215 \cdot 10^{6}$ & $0.2868 \cdot 10^{6}$ & $0.2869 \cdot 10^{6}$ & $0.2882 \cdot 10^{6}$ & $0.2868 \cdot 10^{6}$ \\
$\mathrm{Zn}^{28+}$ & 3.9283 & $0.3308 \cdot 10^{7}$ & $0.1898 \cdot 10^{7}$ & $0.1904 \cdot 10^{7}$ & $0.1692 \cdot 10^{7}$ & $0.1693 \cdot 10^{7}$ & $0.1696 \cdot 10^{7}$ & $0.1691 \cdot 10^{7}$ \\
$\mathrm{Zr}^{38+}$ & 4.2694 & $0.1281 \cdot 10^{8}$ & $0.7356 \cdot 10^{7}$ & $0.7376 \cdot 10^{7}$ & $0.6531 \cdot 10^{7}$ & $0.6536 \cdot 10^{7}$ & $0.6533 \cdot 10^{7}$ & $0.6520 \cdot 10^{7}$ \\
$\mathrm{Sn}^{48+}$ & 4.6519 & $0.3988 \cdot 10^{8}$ & $0.2298 \cdot 10^{8}$ & $0.2304 \cdot 10^{8}$ & $0.2031 \cdot 10^{8}$ & $0.2033 \cdot 10^{8}$ & $0.2028 \cdot 10^{8}$ & $0.2025 \cdot 10^{8}$ \\
$\mathrm{Nd}^{58+}$ & 4.9123 & $0.1103 \cdot 10^{9}$ & $0.6389 \cdot 10^{8}$ & $0.6408 \cdot 10^{8}$ & $0.5622 \cdot 10^{8}$ & $0.5627 \cdot 10^{8}$ & $0.5600 \cdot 10^{8}$ & $0.5593 \cdot 10^{8}$ \\
$\mathrm{Yb}^{68+}$ & 5.3108 & $0.2819 \cdot 10^{9}$ & $0.1646 \cdot 10^{9}$ & $0.1651 \cdot 10^{9}$ & $0.1440 \cdot 10^{9}$ & $0.1442 \cdot 10^{9}$ & $0.1430 \cdot 10^{9}$ & $0.1429 \cdot 10^{9}$ \\
$\mathrm{Hg}^{78+}$ & 5.4648 & $0.7020 \cdot 10^{9}$ & $0.4139 \cdot 10^{9}$ & $0.4154 \cdot 10^{9}$ & $0.3600 \cdot 10^{9}$ & $0.3604 \cdot 10^{9}$ & $0.3561 \cdot 10^{9}$ & $0.3557 \cdot 10^{9}$ \\
$\mathrm{Th}^{88+}$ & 5.7848 & $0.1695 \cdot 10^{10}$ & $0.1012 \cdot 10^{10}$ & $0.1016 \cdot 10^{10}$ & $0.8747 \cdot 10^{9}$ & $0.8758 \cdot 10^{9}$ & $0.8597 \cdot 10^{9}$ & $0.8590 \cdot 10^{9}$ \\
$\mathrm{U}^{90+}$ & 5.8571 & $0.2018 \cdot 10^{10}$ & $0.1208 \cdot 10^{10}$ & $0.1213 \cdot 10^{10}$ & $0.1043 \cdot 10^{10}$ & $0.1044 \cdot 10^{10}$ & $0.1023 \cdot 10^{10}$ & $0.1023 \cdot 10^{10}$
\end{tabular}

Таблица 2. Отдельные вклады в полевой сдвиг в терминах $F$-фактора (в $\mathrm{MHz} / \mathrm{fm}^{2}$ ) для разности энергий $(1 s 2 s)_{J}-(1 s)^{2}$ и $(1 s 2 p)_{J}-(1 s)^{2}$ в гелиеподобных ионах

\begin{tabular}{|c|c|c|c|c|c|c|c|}
\hline И & & & & & & & \\
\hline & $\begin{array}{c}\text { КЭД } \\
\text { Сумма }\end{array}$ & $\begin{array}{r}-0.2 \\
0.0 \\
-0.26\end{array}$ & $\begin{array}{r}-0.2 \\
0.0 \\
-0.26\end{array}$ & $\begin{array}{r}0 . \\
-0.3\end{array}$ & $\begin{array}{r}0 . \\
-0.3\end{array}$ & $\begin{array}{r}0 . \\
-0.3\end{array}$ & $\begin{array}{r}-0.3073 \\
0.0005 \\
-0.3068(\end{array}$ \\
\hline & $\begin{array}{c}\text { КЭД } \\
\text { Сумма }\end{array}$ & $\begin{array}{r}-0.2 \\
0.0 \\
-0.23\end{array}$ & $\begin{array}{r}-0.2 \\
0.0 \\
-0.23\end{array}$ & $\begin{array}{r}-0 . \\
0 . \\
-0.2\end{array}$ & $\begin{array}{r}-0 . \\
0 . \\
-0.2\end{array}$ & $\begin{array}{r}-0.267 \\
0.000 \\
-0.2671\end{array}$ & $\begin{array}{r}-0.2693 \\
0.0007 \\
-0.2686(\end{array}$ \\
\hline & $\begin{array}{l}\text { ез КЭД } \\
\text { КЭД } \\
\text { Сумма }\end{array}$ & $\begin{array}{r}-0.1 \\
0.00 \\
-0.140\end{array}$ & $\begin{array}{r}-0.1 \\
0.0 \\
-0.13\end{array}$ & $\begin{array}{r}-0.1 \\
0.00 \\
-0.16\end{array}$ & $\begin{array}{r}-0.161 \\
0.000 \\
-0.1609\end{array}$ & $\begin{array}{r}-0.161 \\
0.000 \\
-0.1606\end{array}$ & $\begin{array}{r}-0.161 \\
0.000 \\
-0.1612\end{array}$ \\
\hline & $\begin{array}{c}\text { Без КЭД } \\
\text { КЭД } \\
\text { Сумма }\end{array}$ & $\begin{array}{r}-0.5455 \\
0.0023 . \\
-0.5432(2\end{array}$ & $\begin{array}{r}-0.5 \\
0.00 \\
-0.541\end{array}$ & $\begin{array}{r}-0 . \\
0 . \\
-0.62\end{array}$ & $\begin{array}{r}-0.6 \\
0.0 \\
-0.62\end{array}$ & $\begin{array}{r}-0.627 \\
0.002 \\
-0.6251\end{array}$ & $\begin{array}{r}-0.629 \\
0.002 \\
-0.6264\end{array}$ \\
\hline & $\begin{array}{c}\text { Без КЭД } \\
\text { КЭД } \\
\text { Сумма }\end{array}$ & $\begin{array}{r}-0.1691 \cdot 1 \\
0.0008 \cdot 1 \\
-0.1683(2)\end{array}$ & $\begin{array}{r}-0.1684 \\
0.0008 . \\
-0.1676(2\end{array}$ & $\begin{array}{r}-0.195 \\
0.0009 \\
-0.1948\end{array}$ & $\begin{array}{r}-0.1 \\
0.00 \\
-0.19\end{array}$ & $\begin{array}{r}-0.196 \\
0.000 \\
-0.1951\end{array}$ & $\begin{array}{r}-0.196 \\
0.0009 \\
-0.1954(\end{array}$ \\
\hline & $\begin{array}{c}\text { Без КЭД } \\
\text { КЭД } \\
\text { Сумма }\end{array}$ & $\begin{array}{r}-0.4639 \cdot 1 \\
0.0024 \cdot 1 \\
-0.4615(5)\end{array}$ & $\begin{array}{c}-0.4620 \cdot 10^{8} \\
0.0024 \cdot 10^{8} \\
-0.4596(5) \cdot 10^{8}\end{array}$ & $\begin{array}{r}-0.5406 \\
0.0029 \\
-0.5377(5\end{array}$ & $\begin{array}{r}-0.5401 \\
0.0029 \\
-0.5372(\end{array}$ & $\begin{array}{r}-0.5428 \\
0.0029 \\
-0.5399\end{array}$ & $\begin{array}{r}0.002 \\
-0.5406\end{array}$ \\
\hline & $\begin{array}{c}\text { Без КЭД } \\
\text { КЭД } \\
\text { Сумма }\end{array}$ & $\begin{array}{r}-0.1173 \cdot 1 \\
0.0007 \cdot 1 \\
-0.1166(2)\end{array}$ & $\begin{array}{r}-0.1168 \\
0.0007 \\
-0.1161(2\end{array}$ & $\begin{array}{r}-0.137 \\
0.000 \\
-0.1371\end{array}$ & $\begin{array}{r}-0.137 \\
0.000 \\
-0.1369\end{array}$ & $\begin{array}{r}-0.138 \\
0.000 \\
-0.1381\end{array}$ & $\begin{array}{r}-0.139 \\
0.000 \\
-0.1382\end{array}$ \\
\hline & $\begin{array}{c}\text { Без КЭД } \\
\text { КЭД } \\
\text { Сумма }\end{array}$ & $\begin{array}{r}-0.2881 \cdot \\
0.0016 \cdot 1 \\
-0.2865(4)\end{array}$ & $\begin{array}{r}-0.2866 \\
0.0016 \\
-0.2850(4\end{array}$ & $\begin{array}{r}-0.3 \\
0.00 \\
-0.340\end{array}$ & $\begin{array}{r}-0.341 \\
0.001 \\
-0.3397\end{array}$ & $\begin{array}{r}-0.345 \\
0.0019 \\
-0.3439\end{array}$ & $\begin{array}{c}-0.3463 \cdot 10^{9} \\
0.0019 \cdot 10^{9} \\
-0.3444(5) \cdot 10^{9}\end{array}$ \\
\hline $\mathrm{T}$ & $\begin{array}{c}\text { Без КЭД } \\
\text { КЭД } \\
\text { Сумма }\end{array}$ & \begin{tabular}{|c|}
$-0.6832 \cdot 10^{9}$ \\
$0.0037 \cdot 10^{9}$ \\
$-0.6795(10) \cdot 10^{9}$
\end{tabular} & \begin{tabular}{|c|}
$-0.6789 \cdot 10^{9}$ \\
$0.0037 \cdot 10^{9}$ \\
$-0.6752(10) \cdot 10^{9}$
\end{tabular} & \begin{tabular}{|c|}
$-0.8204 \cdot 10^{9}$ \\
$0.0045 \cdot 10^{9}$ \\
$-0.8159(12) \cdot 10^{9}$
\end{tabular} & $\begin{array}{c}-0.8194 \cdot 10^{9} \\
0.0045 \cdot 10^{9} \\
-0.8149(12) \cdot 10^{9}\end{array}$ & $\begin{array}{c}-0.8354 \cdot 10^{9} \\
0.0045 \cdot 10^{9} \\
-0.8309(12) \cdot 10^{9}\end{array}$ & $\begin{array}{c}-0.8361 \cdot 10^{9} \\
0.0045 \cdot 10^{9} \\
-0.8316(12) \cdot 10^{9}\end{array}$ \\
\hline $\mathrm{U}^{90+}$ & $\begin{array}{c}\text { КЭД } \\
\text { Сумма }\end{array}$ & $\begin{array}{c}-0.8099 \cdot 10^{9} \\
0.0044 \cdot 10^{9} \\
-0.8055(15) \cdot 10^{9}\end{array}$ & $\begin{array}{c}-0.8046 \cdot 10^{9} \\
0.0044 \cdot 10^{9} \\
-0.8002(15) \cdot 10^{9}\end{array}$ & $\begin{array}{c}-0.9749 \cdot 10^{9} \\
0.0053 \cdot 10^{9} \\
-0.9696(18) \cdot 10^{9}\end{array}$ & $\begin{array}{c}-0.9737 \cdot 10^{9} \\
0.0053 \cdot 10^{9} \\
-0.9684(18) \cdot 10^{9}\end{array}$ & $\begin{array}{c}-0.9945 \cdot 10^{9} \\
0.0053 \cdot 10^{9} \\
-0.9892(18) \cdot 10^{9}\end{array}$ & $\begin{array}{c}-0.9953 \cdot 10^{9} \\
0.0053 \cdot 10^{9} \\
-0.9900(18) \cdot 10^{9}\end{array}$ \\
\hline
\end{tabular}


Таблица 3. Массовый сдвиг в терминах $K$-фактора (в 1000 GHz-a.m.u.) для состояний $1 s^{2},(1 s 2 s)_{J}$ и $(1 s 2 p)_{J}$ гелиеподобных ионов в интервале $Z=12-50$

\begin{tabular}{|c|c|c|c|c|}
\hline$Z$ & Состояние & Без КЭД & КЭД & Сумма \\
\hline 12 & $\begin{array}{c}(1 s)^{2} \\
(1 s 2 s)_{0} \\
(1 s 2 s)_{1} \\
\left(1 s 2 p_{1 / 2}\right)_{0} \\
\left(1 s 2 p_{1 / 2}\right)_{1} \\
\left(1 s 2 p_{3 / 2}\right)_{1} \\
\left(1 s 2 p_{3 / 2}\right)_{2}\end{array}$ & $\begin{array}{l}498.41 \\
315.76 \\
317.39 \\
281.09 \\
281.17 \\
347.47 \\
280.98\end{array}$ & $\begin{array}{l}0.82 \\
0.47 \\
0.47 \\
0.41 \\
0.41 \\
0.41 \\
0.41\end{array}$ & $\begin{array}{c}499.23(7) \\
316.23(3) \\
317.87(3) \\
281.49(3) \\
281.58(3) \\
347.88(3) \\
281.38(3)\end{array}$ \\
\hline 20 & $\begin{array}{c}(1 s)^{2} \\
(1 s 2 s)_{0} \\
(1 s 2 s)_{1} \\
\left(1 s 2 p_{1 / 2}\right)_{0} \\
\left(1 s 2 p_{1 / 2}\right)_{1} \\
\left(1 s 2 p_{3 / 2}\right)_{1} \\
\left(1 s 2 p_{3 / 2}\right)_{2}\end{array}$ & $\begin{array}{c}1407.36 \\
887.50 \\
890.48 \\
785.66 \\
791.03 \\
978.72 \\
784.65\end{array}$ & $\begin{array}{l}9.51 \\
5.49 \\
5.49 \\
4.73 \\
4.72 \\
4.72 \\
4.73\end{array}$ & $\begin{array}{c}1416.9(4) \\
893.0(2) \\
896.0(2) \\
790.4(2) \\
795.8(2) \\
983.4(2) \\
789.4(2)\end{array}$ \\
\hline 30 & $\begin{array}{c}(1 s)^{2} \\
(1 s 2 s)_{0} \\
(1 s 2 s)_{1} \\
\left(1 s 2 p_{1 / 2}\right)_{0} \\
\left(1 s 2 p_{1 / 2}\right)_{1} \\
\left(1 s 2 p_{3 / 2}\right)_{1} \\
\left(1 s 2 p_{3 / 2}\right)_{2}\end{array}$ & $\begin{array}{l}3190.87 \\
2010.21 \\
2015.14 \\
1777.18 \\
1839.49 \\
2170.82 \\
1771.53\end{array}$ & $\begin{array}{l}66.97 \\
38.84 \\
38.84 \\
33.43 \\
33.42 \\
33.34 \\
33.33\end{array}$ & $\begin{array}{c}3258(2) \\
2049(1) \\
2054(1) \\
1811(1) \\
1873(1) \\
2204(1) \\
1805(1)\end{array}$ \\
\hline 40 & $\begin{array}{c}(1 s)^{2} \\
(1 s 2 s)_{0} \\
(1 s 2 s)_{1} \\
\left(1 s 2 p_{1 / 2}\right)_{0} \\
\left(1 s 2 p_{1 / 2}\right)_{1} \\
\left(1 s 2 p_{3 / 2}\right)_{1} \\
\left(1 s 2 p_{3 / 2}\right)_{2}\end{array}$ & $\begin{array}{l}5689.51 \\
3587.88 \\
3595.20 \\
3175.61 \\
3367.14 \\
3789.04 \\
3156.52\end{array}$ & $\begin{array}{l}272.21 \\
158.64 \\
158.64 \\
136.50 \\
136.52 \\
135.82 \\
135.81\end{array}$ & $\begin{array}{c}5962(7) \\
3747(4) \\
3754(3) \\
3312(3) \\
3504(3) \\
3925(3) \\
3292(3)\end{array}$ \\
\hline 50 & $\begin{array}{c}(1 s)^{2} \\
(1 s 2 s)_{0} \\
(1 s 2 s)_{1} \\
\left(1 s 2 p_{1 / 2}\right)_{0} \\
\left(1 s 2 p_{1 / 2}\right)_{1} \\
\left(1 s 2 p_{3 / 2}\right)_{1} \\
\left(1 s 2 p_{3 / 2}\right)_{2}\end{array}$ & $\begin{array}{l}8891.04 \\
5619.61 \\
5630.06 \\
4987.25 \\
5346.36 \\
5856.12 \\
4937.86\end{array}$ & $\begin{array}{l}829.61 \\
486.12 \\
486.12 \\
418.33 \\
418.45 \\
415.02 \\
414.89\end{array}$ & $\begin{array}{c}9721(17) \\
6106(9) \\
6116(9) \\
5406(8) \\
5765(8) \\
6271(8) \\
5353(8)\end{array}$ \\
\hline
\end{tabular}

релятивистского оператора отдачи $[21,22,29]$, который имеет вид

$$
\begin{aligned}
H_{M}= & \frac{1}{2 M} \sum_{i, k}\left[\mathbf{p}_{i} \cdot \mathbf{p}_{k}\right. \\
& \left.-\frac{\alpha Z}{r_{i}}\left(\boldsymbol{\alpha}_{i}+\frac{\left(\boldsymbol{\alpha}_{i} \cdot \mathbf{r}_{i}\right) \mathbf{r}_{i}}{r_{i}^{2}}\right) \cdot \mathbf{p}_{k}\right] .
\end{aligned}
$$

Здесь суммирование идет по всем атомным электронам. В настоящее время этот оператор широко используется в атомных расчетах [8-10,14,15,30-33]. Расчеты обычно выполняются посредством усреднения оператора (11) с волновыми функциями, полученными из гамильтониана ДКБ. Такой подход позволяет в принципе учесть корреляционные поправки к эффекту отдачи во всех порядках
Таблица 4. Массовый сдвиг в терминах $K$-фактора (в 1000 $\mathrm{GHz}$-a.m.u.) для состояний $1 s^{2},(1 s 2 s)_{J}$ и $(1 s 2 p)_{J}$ гелиеподоб-

\begin{tabular}{|c|c|c|c|c|}
\hline$Z$ & Состояние & Без КЭД & КЭД & Сумма \\
\hline 60 & $\begin{array}{c}(1 s)^{2} \\
(1 s 2 s)_{0} \\
(1 s 2 s)_{1} \\
\left(1 s 2 p_{1 / 2}\right)_{0} \\
\left(1 s 2 p_{1 / 2}\right)_{1} \\
\left(1 s 2 p_{3 / 2}\right)_{1} \\
\left(1 s 2 p_{3 / 2}\right)_{2}\end{array}$ & $\begin{array}{l}12764 \\
8096 \\
8110 \\
7213 \\
7761 \\
8366 \\
7105\end{array}$ & $\begin{array}{l}2133 \\
1257 \\
1257 \\
1082 \\
1082 \\
1069 \\
1069\end{array}$ & $\begin{array}{l}14.897(37) \cdot 10^{3} \\
9.353(21) \cdot 10^{3} \\
9.368(21) \cdot 10^{3} \\
8.296(19) \cdot 10^{3} \\
8.844(18) \cdot 10^{3} \\
9.435(17) \cdot 10^{3} \\
8.174(17) \cdot 10^{3}\end{array}$ \\
\hline 70 & $\begin{array}{c}(1 s)^{2} \\
(1 s 2 s)_{0} \\
(1 s 2 s)_{1} \\
\left(1 s 2 p_{1 / 2}\right)_{0} \\
\left(1 s 2 p_{1 / 2}\right)_{1} \\
\left(1 s 2 p_{3 / 2}\right)_{1} \\
\left(1 s 2 p_{3 / 2}\right)_{2}\end{array}$ & $\begin{array}{c}17216 \\
10974 \\
10994 \\
9830 \\
10580 \\
11273 \\
9618\end{array}$ & $\begin{array}{l}4945 \\
2936 \\
2936 \\
2528 \\
2528 \\
2483 \\
2483\end{array}$ & $\begin{array}{l}22.160(74) \cdot 10^{3} \\
13.910(43) \cdot 10^{3} \\
13.930(42) \cdot 10^{3} \\
12.358(38) \cdot 10^{3} \\
13.108(36) \cdot 10^{3} \\
13.756(35) \cdot 10^{3} \\
12.100(35) \cdot 10^{3}\end{array}$ \\
\hline 80 & $\begin{array}{c}(1 s)^{2} \\
(1 s 2 s)_{0} \\
(1 s 2 s)_{1} \\
\left(1 s 2 p_{1 / 2}\right)_{0} \\
\left(1 s 2 p_{1 / 2}\right)_{1} \\
\left(1 s 2 p_{3 / 2}\right)_{1} \\
\left(1 s 2 p_{3 / 2}\right)_{2}\end{array}$ & $\begin{array}{l}22032 \\
14143 \\
14172 \\
12760 \\
13715 \\
14467 \\
12375\end{array}$ & $\begin{array}{l}10783 \\
6456 \\
6456 \\
5561 \\
5561 \\
5422 \\
5422\end{array}$ & $\begin{array}{c}32.82(15) \cdot 10^{3} \\
20.60(9) \cdot 10^{3} \\
20.63(9) \cdot 10^{3} \\
18.32(8) \cdot 10^{3} \\
19.28(8) \cdot 10^{3} \\
19.89(8) \cdot 10^{3} \\
17.80(8) \cdot 10^{3}\end{array}$ \\
\hline 90 & $\begin{array}{c}(1 s)^{2} \\
(1 s 2 s)_{0} \\
(1 s 2 s)_{1} \\
\left(1 s 2 p_{1 / 2}\right)_{0} \\
\left(1 s 2 p_{1 / 2}\right)_{1} \\
\left(1 s 2 p_{3 / 2}\right)_{1} \\
\left(1 s 2 p_{3 / 2}\right)_{2}\end{array}$ & $\begin{array}{l}26582 \\
17243 \\
17281 \\
15717 \\
16870 \\
17623 \\
15067\end{array}$ & $\begin{array}{l}22835 \\
13809 \\
13809 \\
11897 \\
11897 \\
11488 \\
11488\end{array}$ & $\begin{array}{l}49.42(39) \cdot 10^{3} \\
31.05(26) \cdot 10^{3} \\
31.09(26) \cdot 10^{3} \\
27.61(25) \cdot 10^{3} \\
28.77(24) \cdot 10^{3} \\
29.11(24) \cdot 10^{3} \\
26.56(24) \cdot 10^{3}\end{array}$ \\
\hline 92 & $\begin{array}{c}(1 s)^{2} \\
(1 s 2 s)_{0} \\
(1 s 2 s)_{1} \\
\left(1 s 2 p_{1 / 2}\right)_{0} \\
\left(1 s 2 p_{1 / 2}\right)_{1} \\
\left(1 s 2 p_{3 / 2}\right)_{1} \\
\left(1 s 2 p_{3 / 2}\right)_{2}\end{array}$ & $\begin{array}{l}27357 \\
17792 \\
17833 \\
16262 \\
17453 \\
18197 \\
15546\end{array}$ & $\begin{array}{l}26495 \\
16059 \\
16059 \\
13837 \\
13837 \\
13329 \\
13330\end{array}$ & $\begin{array}{l}53.85(52) \cdot 10^{3} \\
33.85(35) \cdot 10^{3} \\
33.89(35) \cdot 10^{3} \\
30.10(34) \cdot 10^{3} \\
31.29(34) \cdot 10^{3} \\
31.53(33) \cdot 10^{3} \\
28.88(33) \cdot 10^{3}\end{array}$ \\
\hline
\end{tabular}
ных ионов в интервале $Z=60-92$

по $1 / Z$. Следует однако иметь ввиду, что в случае тяжелых многозарядных ионов КЭД вклады отдачи ядра, которые не учитываются оператором (11), могут быть даже больше, чем вклад от этого оператора $[8,27,30]$.

Наконец, кроме отмеченных выше главных вкладов в изотопические сдвиги, в случае тяжелых ионов следует учитывать такие тонкие эффекты, как эффект поляризации ядра и эффект деформации ядра. Первый из этих эффектов обусловлен влиянием электронов на ядерную структуру, которая не является абсолютно жесткой. В результате такого влияния ядро немного деформируется и, как следствие этого, меняется создаваемый им потенциал. Такое изменение сказывается на энергии связи электрона с ядром. В терминах 
Таблица 5. Отдельные вклады в изотопические сдвиги разности энергий $(1 s 2 s)_{J=0,1}-(1 s)^{2}{ }_{\text {в }}{ }^{232,230} \mathrm{Th}^{86+},{ }^{238,236} \mathrm{U}^{88+}{ }^{238,234} \mathrm{U}^{88+}$ $($ в $\mathrm{meV})$ для заданных значений $\delta\left\langle r^{2}\right\rangle$; погрешности величин $\delta\left\langle r^{2}\right\rangle$ не учитываются

\begin{tabular}{|c|c|c|c|}
\hline & $\begin{array}{c}{ }^{232,230} \mathrm{Th}^{86+} \\
{ }^{232,230} \delta\left\langle r^{2}\right\rangle=0.2050 \mathrm{fm}^{2}\end{array}$ & $\begin{array}{c}238,236 \\
{ }^{838+} \\
{ }^{238,236} \delta\left\langle r^{2}\right\rangle=0.1676 \mathrm{fm}^{2}\end{array}$ & $\begin{array}{c}238,234 \mathrm{U}^{88+} \\
238,234\end{array}\left\langle r^{2}\right\rangle=0.334 \mathrm{fm}^{2}$ \\
\hline \multicolumn{4}{|l|}{$(1 s 2 s)_{0}-(1 s)^{2}$} \\
\hline $\begin{array}{c}\text { Главные вклады } \\
\text { Полевой сдвиг } \\
\text { Массовый сдвиг } \\
\text { Сумма ПС и МС }\end{array}$ & $\begin{array}{c}-579 \\
1 \\
-578\end{array}$ & $\begin{array}{c}-561 \\
1 \\
-560\end{array}$ & $\begin{array}{c}-1119 \\
3 \\
-1116\end{array}$ \\
\hline $\begin{array}{c}\text { КЭД поправки } \\
\text { Полевой сдвиг } \\
\text { Массовый сдвиг }\end{array}$ & $\begin{array}{l}3 \\
1\end{array}$ & $\begin{array}{l}3 \\
2\end{array}$ & $\begin{array}{l}6 \\
3\end{array}$ \\
\hline $\begin{array}{l}\text { Другие поправки } \\
\text { Поляризация ядра } \\
\text { Деформация ядра }\end{array}$ & $\begin{array}{l}8 \\
7\end{array}$ & $\begin{array}{c}5 \\
-11\end{array}$ & $\begin{array}{c}11 \\
-12\end{array}$ \\
\hline Полное значение & $-558(11)$ & $-561(15)$ & $-1108(16)$ \\
\hline \multicolumn{4}{|l|}{$(1 s 2 s)_{1}-(1 s)^{2}$} \\
\hline $\begin{array}{c}\text { Главные вклады } \\
\text { Полевой сдвиг } \\
\text { Массовый сдвиг } \\
\text { Сумма ПС и МС }\end{array}$ & $\begin{array}{l}-576 \\
1 \\
-574\end{array}$ & $\begin{array}{l}-558 \\
1 \\
-556\end{array}$ & $\begin{array}{c}-1111 \\
3 \\
-1109\end{array}$ \\
\hline $\begin{array}{c}\text { КЭД поправки } \\
\text { Полевой сдвиг } \\
\text { Массовый сдвиг }\end{array}$ & $\begin{array}{l}3 \\
1 \\
\end{array}$ & $\begin{array}{l}3 \\
2 \\
\end{array}$ & $\begin{array}{l}6 \\
3 \\
\end{array}$ \\
\hline $\begin{array}{l}\text { Другие поправки } \\
\text { Поляризация ядра } \\
\text { Деформация ядра }\end{array}$ & $\begin{array}{l}8 \\
7 \\
\end{array}$ & $\begin{array}{c}5 \\
-11 \\
\end{array}$ & $\begin{array}{c}11 \\
-12 \\
\end{array}$ \\
\hline Полное значение & $-555(11)$ & $-558(15)$ & $-1100(16)$ \\
\hline
\end{tabular}

фейнмановских диаграмм данный эффект описывается диаграммами двухфотонного обмена между электроном и ядром, в которых промежуточные состояния ядра являются возбужденными. Для тяжелых ионов этот эффект вычислялся в работах [34-37].

Под эффектом ядерной деформации подразумевается отличие распределения заряда ядра от сферически симметричной фермиевской модели, задаваемой формулой (6). Для оценки этого эффекта необходимо заменить сферически симметричное фермиевское распределение (6) на следующее [38]:

$$
\rho(r)=\frac{1}{4 \pi} \int d \mathbf{n} \rho(\mathbf{r}),
$$

где $\rho(\mathbf{r})$ - аксиально-симметричное распределение Ферми:

$$
\rho(\mathbf{r})=\frac{N}{1+\exp \left[\left(r-r_{0}\left(1+\beta_{20} Y_{20}(\theta)+\beta_{40} Y_{40}(\theta)\right) / a\right]\right.},
$$

удовлетворяющее нормировочному условию:

$$
\int d \mathbf{r} \rho(\mathbf{r})=1
$$

Здесь $Y_{20}(\theta)$ и $Y_{40}(\theta)$ - сферические функции, $\beta_{20}$ и $\beta_{40}$ - параметры квадрупольной и гексадекапольной деформаций [38-41]. Разность атомных уровней энергии, вычисленных для деформированного распределения (12) и стандартного сферически симметричного распределения Ферми (6) при одинаковом среднеквадратичном зарядовом радиусе, определяет эффект ядерной деформации. В тяжелых ионах этот эффект оценивался, например, в работе [38].

\section{3. Результаты и обсуждение}

В табл. 1 приведены результаты вычислений $F$-фактора полевого сдвига в широком диапазоне значений заряда ядра $Z$. Вычисления выполнены в рамках уравнения ДКБ методом наложения конфигураций в базисе функций Дирака-Фока-Штурма. В табл. 2 приведены соот- 
Таблица 6. Отдельные вклады в изотопические сдвиги разности энергий $(1 s 2 p)_{J=0,2}-(1 s)^{2}{ }_{\text {в }}{ }^{232,230} \mathrm{Th}^{86+},{ }^{238,236} \mathrm{U}^{88+}{ }^{238,234} \mathrm{U}^{88+}$ $($ в $\mathrm{meV})$ для заданных значений $\delta\left\langle r^{2}\right\rangle$; погрешности величин $\delta\left\langle r^{2}\right\rangle$ не учитываются

\begin{tabular}{|c|c|c|c|}
\hline & $\begin{array}{c}{ }^{232,230} \mathrm{Th}^{86+} \\
{ }^{232,230} \delta\left\langle r^{2}\right\rangle=0.2050 \mathrm{fm}^{2}\end{array}$ & $\begin{array}{c}{ }^{238,236} \mathrm{U}^{88+} \\
{ }^{238,236} \delta\left\langle r^{2}\right\rangle=0.1676 \mathrm{fm}^{2}\end{array}$ & $\begin{array}{c}{ }^{238,234} \mathrm{U}^{88+} \\
238,234 \\
\delta\left\langle r^{2}\right\rangle=0.334 \mathrm{fm}^{2}\end{array}$ \\
\hline \multicolumn{4}{|l|}{$\left(1 s 2 p_{1 / 2}\right)_{0}-(1 s)^{2}$} \\
\hline $\begin{array}{c}\text { Главные вклады } \\
\text { Полевой сдвиг } \\
\text { Массовый сдвиг } \\
\text { Сумма ПС и МС }\end{array}$ & $\begin{array}{l}-696 \\
2 \\
-694\end{array}$ & $\begin{array}{l}-676 \\
2 \\
-674\end{array}$ & $\begin{array}{c}-1347 \\
3 \\
-1343\end{array}$ \\
\hline $\begin{array}{c}\text { КЭД поправки } \\
\text { Полевой сдвиг } \\
\text { Массовый сдвиг }\end{array}$ & $\begin{array}{l}4 \\
2\end{array}$ & $\begin{array}{l}4 \\
2\end{array}$ & $\begin{array}{l}7 \\
4\end{array}$ \\
\hline $\begin{array}{l}\text { Другие поправки } \\
\text { Поляризация ядра } \\
\text { Деформация ядра }\end{array}$ & $\begin{array}{c}10 \\
9\end{array}$ & $\begin{array}{c}6 \\
-13 \\
\end{array}$ & $\begin{array}{c}14 \\
-14\end{array}$ \\
\hline Полное значение & $-669(13)$ & $-675(18)$ & $-1332(19)$ \\
\hline \multicolumn{4}{|l|}{$\left(1 s 2 p_{3 / 2}\right)_{2}-(1 s)^{2}$} \\
\hline $\begin{array}{c}\text { Главные вклады } \\
\text { Полевой сдвиг } \\
\text { Массовый сдвиг } \\
\text { Сумма ПС и МС }\end{array}$ & $\begin{array}{l}-709 \\
2 \\
-707 \\
\end{array}$ & $\begin{array}{l}-690 \\
2 \\
-688 \\
\end{array}$ & $\begin{array}{c}-1375 \\
4 \\
-1371 \\
\end{array}$ \\
\hline $\begin{array}{c}\text { КЭД поправки } \\
\text { Полевой сдвиг } \\
\text { Массовый сдвиг }\end{array}$ & $\begin{array}{l}4 \\
2\end{array}$ & $\begin{array}{l}4 \\
2\end{array}$ & $\begin{array}{l}7 \\
4\end{array}$ \\
\hline $\begin{array}{c}\text { Другие поправки } \\
\text { Поляризация ядра } \\
\text { Деформация ядра }\end{array}$ & $\begin{array}{c}10 \\
9\end{array}$ & $\begin{array}{c}6 \\
-13\end{array}$ & $\begin{array}{c}14 \\
-14\end{array}$ \\
\hline Полное значение & $-682(13)$ & $-689(19)$ & $-1360(19)$ \\
\hline
\end{tabular}

ветствующие значения для разности энергий однократно возбужденных и основного состояний. Здесь, помимо основного вклада из табл. 1, учтены также КЭД поправки, вычисленные посредством использования аналитических формул для водородоподобных ионов из работ $[19,20]$. Это было сделано так же, как и в расчетах соответствующих поправок в работе [8]. А именно $\Delta_{s}$-фактор, описывающий КЭД поправку к эффекту конечности размера ядра для $s$-состояний водородоподобного иона [19], умножался на вклад эффекта конечности размера ядра в энергию рассматриваемого перехода в двухэлектронном ионе. Значения среднеквадратичных зярядовых радиусов ядер были взяты из [42]. Оценка погрешностей была выполнена по аналогии с работой [8].

В табл. 3, 4 приведены значения $K$-фактора массового сдвига. Значения приведены как без учета КЭД вклада, так и с учетом КЭД вклада. Эти величины получены из прецизионных расчетов, выполненных по теории возмущений в работе [27]. В то время как в случае одиночных уровней значения $K$-фактора могут быть получены из данных работы [27] простым умножением на массу соответствующего изотопа, в случае квазивырожденных уровней $\left(1 s 2 p_{1 / 2}\right)_{1}$ и $\left(1 s 2 p_{3 / 2}\right)_{1}$ требуется диагонализовать оператор полной энергии иона в пространстве рассматриваемых квазивырожденных состояний. В первом порядке по $m / M$ интересующая нас поправка на отдачу ядра определяется формулой:

$$
\Delta E=\frac{V_{11}+V_{22}}{2} \pm \frac{1}{2} \frac{\left(H_{11}-H_{22}\right)\left(V_{11}-V_{22}\right)+4 H_{12} V_{12}}{\sqrt{\left(H_{11}-H_{22}\right)^{2}+4 H_{12}^{2}}},
$$

где $V_{i k}$ - матричные элементы полной поправки на отдачу ядра в пространстве невозмущенных квазивырожденных состояний $\left(1 s 2 p_{1 / 2}\right)_{1}$ и $\left(1 s 2 p_{3 / 2}\right)_{1}, H_{i k}-$ матричные элементы полного оператора энергии (за исключением поправок на отдачу) в рассматриваемом пространстве. Значения $V_{i k}$, которые включают релятивистские, корреляционные и КЭД вклады, были взяты из работы [27], а значения $H_{i k}$ - из работ [11-13]. Как видно из таблиц, значения КЭД вклада очень быстро растут с ростом $Z$ и для $Z=92$ практически удваивают полное значение массового сдвига. Погрешности определяются в основном двумя факторами: приближенным учетом поправки на конечность размера ядра к КЭД вкладу 
Таблица 7. Отдельные вклады в изотопические сдвиги разности энергий $(1 s 2 p)_{J=1}-(1 s)^{2}$ в ${ }^{232,230} \mathrm{Th}^{86+},{ }^{238,236} \mathrm{U}^{88+}{ }^{238,234} \mathrm{U}^{88+}$ $($ в $\mathrm{meV})$ для заданных значений $\delta\left\langle r^{2}\right\rangle$; погрешности величин $\delta\left\langle r^{2}\right\rangle$ не учитываются

\begin{tabular}{|c|c|c|c|}
\hline & $\begin{array}{c}{ }^{232,230} \mathrm{Th}^{86+} \\
232,230 \\
\delta\left\langle r^{2}\right\rangle=0.2050 \mathrm{fm}^{2}\end{array}$ & $\begin{array}{c}{ }^{238,236} \mathrm{U}^{88+} \\
{ }^{238,236} \delta\left\langle r^{2}\right\rangle=0.1676 \mathrm{fm}^{2}\end{array}$ & 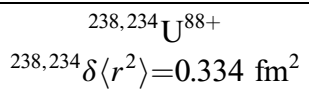 \\
\hline \multicolumn{4}{|l|}{$\left(1 s 2 p_{1 / 2}\right)_{1}-(1 s)^{2}$} \\
\hline $\begin{array}{c}\text { Главные вклады } \\
\text { Полевой сдвиг } \\
\text { Массовый сдвиг } \\
\text { Сумма ПС и МС }\end{array}$ & $\begin{array}{l}-695 \\
2 \\
-693\end{array}$ & $\begin{array}{l}-675 \\
1 \\
-673\end{array}$ & $\begin{array}{c}-1345 \\
3 \\
-1342\end{array}$ \\
\hline $\begin{array}{c}\text { КЭД поправки } \\
\text { Полевой сдвиг } \\
\text { Массовый сдвиг }\end{array}$ & $\begin{array}{l}4 \\
2\end{array}$ & $\begin{array}{l}4 \\
2\end{array}$ & $\begin{array}{l}7 \\
4\end{array}$ \\
\hline $\begin{array}{l}\text { Другие поправки } \\
\text { Поляризация ядра } \\
\text { Деформация ядра }\end{array}$ & $\begin{array}{c}10 \\
9\end{array}$ & $\begin{array}{c}6 \\
-13 \\
\end{array}$ & $\begin{array}{c}14 \\
-14\end{array}$ \\
\hline Полное значение & $-669(13)$ & $-675(18)$ & $-1331(19)$ \\
\hline \multicolumn{4}{|l|}{$\left(1 s 2 p_{3 / 2}\right)_{1}-(1 s)^{2}$} \\
\hline $\begin{array}{c}\text { Главные вклады } \\
\text { Полевой сдвиг } \\
\text { Массовый сдвиг } \\
\text { Сумма ПС и МС }\end{array}$ & $\begin{array}{l}-708 \\
1 \\
-707\end{array}$ & $\begin{array}{l}-689 \\
1 \\
-688\end{array}$ & $\begin{array}{l}-1374 \\
3 \\
-1371\end{array}$ \\
\hline $\begin{array}{c}\text { КЭД поправки } \\
\text { Полевой сдвиг } \\
\text { Массовый сдвиг }\end{array}$ & $\begin{array}{l}4 \\
2\end{array}$ & $\begin{array}{l}4 \\
2\end{array}$ & $\begin{array}{l}7 \\
4\end{array}$ \\
\hline $\begin{array}{l}\text { Другие поправки } \\
\text { Поляризация ядра } \\
\text { Деформация ядра }\end{array}$ & $\begin{array}{c}10 \\
9\end{array}$ & $\begin{array}{c}6 \\
-13 \\
\end{array}$ & $\begin{array}{c}14 \\
-14\end{array}$ \\
\hline Полное значение & $-682(13)$ & $-689(19)$ & $-1360(19)$ \\
\hline
\end{tabular}

в эффект отдачи и невычисленными КЭД поправками первого порядка по $1 / Z[27]$.

В табл. 5-7 приводятся полные теоретические значения изотопических сдвигов разностей энергий однократно возбужденных и основного состояний гелиеподобных ионов тория и урана. Кроме полевого и массового сдвигов, здесь также учтены вклады от поляризации и деформации ядер. Именно эти вклады, главным образом, и определяют полную теоретическую погрешность. Отметим, что эта погрешность превышает ошибку, происходящую от использования приближенной формулы (8) вместо точного расчета разности энергий связи для двух разных изотопов. Тем не менее следует помнить, что прямой расчет может быть важен в случае, когда разность ядерных радиусов является достаточно большой.

\section{4. Заключение}

В настоящей работе проведены прецизионные расчеты изотопических сдвигов уровней энергии гелиеподобных многозарядных ионов. Расчеты включают релятивистские и корреляционные вклады в полевой и массовый сдвиги, а также соответствующие КЭД эффекты. Для изотопов тория и урана учтены также эффекты поляризации и деформации ядер. В результате проведенных расчетов получены наиболее точные теоретические значения $F$ - и $K$-факторов в широком диапазоне заряда ядра $Z=12-92$, а в случае гелиеподобных ионов тория и урана - полные значения изотопических сдвигов с учетом поправок на поляризацию и деформацию ядер.

\section{Финансирование работы}

Работа выполнена при финансовой поддержке Российского научного фонда (грант № 18-32-00275). Работа М.Ю.К., А.В.М., Р.В.П., В.М.Ш. поддержана Фондом развития теоретической физики и математики „БАЗИС“.

\section{Конфликт интересов}

Авторы заявляют, что у них нет конфликта интересов. 


\section{Список литературы}

[1] Elliott S.R., Beiersdorfer P., Chen M.H. // Phys. Rev. Lett. 1996. V. 76. P. 1031; Erratum: Phys. Rev. Lett. 1996. V. 77. P. 4278. doi 10.1103/PhysRevLett.76.1031

[2] Elliott S.R., Beiersdorfer P., Chen M.H., Decaux V., Knapp D.A. // Phys. Rev. C. 1998. V. 57. P. 583. doi 10.1103/PhysRevC.57.583

[3] Schuch R., Lindroth E., Madzunkov S., Fogle M., Mohamed T., Indelicato P. // Phys. Rev. Lett. 2005. V. 95. P. 183003. doi 10.1103/PhysRevLett.95.183003

[4] Soria Orts R., Harman Z., Crespo Lopez-Urrutia J.R., Artemyev A.N., Bruhns H., Gonzalez Martinez A.J., Jentschura U.D., Keitel C.H., Lapierre A., Mironov V., Shabaev V.M., Tawara H., Tupitsyn I.I., Ullrich J., Volotka A.V. // Phys. Rev. Lett. 2006. V. 97. P. 103002. doi 10.1103/PhysRevLett.97.103002

[5] Brandau C., Kozhuharov C., Harman Z., Müller A., Schippers S., Kozhedub Y. S., Bernhardt D., Böhm S., Jacobi J., Schmidt E.W., Mokler P.H., Bosch F., Kluge H.J., Stöhlker Th., Beckert K., Beller P., Nolden F., Steck M., Gumberidze A., Reuschl R., Spillmann U., Currell F.J, Tupitsyn I.I., Shabaev V.M., Jentschura U.D., Keitel C.H., Wolf A., Stachura Z. // Phys. Rev. Lett. 2008. V. 100. P. 073201. doi 10.1103/PhysRevLett.100.073201

[6] Brandau C., Kozhuharov C., Müller A., Bernhardt D., Bosch F., Boutin D., Currell F.J., Dimopoulou C., Franzke B., Fritzsche S., Gumberidze A., Harman Z., Jentschura U.D., Keitel C.H., Kozhedub Y.S., Krücken R., Litvinov Yu.A., Nolden F., O'Rourke B., Reuschl R., Schippers S., Shabaev V.M., Spillmann U., Stachura Z., Steck M., Stöhlker Th., Tupitsyn I.I., Winters D.F.A., Wolf A. // Hyperfine Interact. 2010. V. 196. P. 115. doi 10.1007/s10751009-0142-2

[7] Brandau C., Kozhuharov C., Müller A., Bernhardt D., Banas D., Bosch F., Currell F.J., Dimopoulou C., Gumberidze A., Hagmann S., Hillenbrand P.-M., Heil M., Lestinsky M., Litvinov Yu.A, Martin R., Nolden F., Reuschl R., Sanjari S., Schippers S., Schneider D., Shubina D., Simon H., Spillmann U., Stachura Z., Steck M., Stöhlker Th., Weber G., Wiedeking M., Winckler N., Winters D.F.A. // Phys. Scr. T. 2013. V. 156. P. 014050. doi 10.1088/00318949/2013/T156/014050

[8] Zubova N.A., Kozhedub Y.S., Shabaev V.M., Tupitsyn I.I., Volotka A.V., Plunien G., Brandau C., Stöhlker Th. // Phys. Rev. A. 2014. V. 90. P. 062512. doi 10.1103/PhysRevA.90.062512

[9] Zubova N.A, Malyshev A.V., Tupitsyn I.I., Shabaev V.M., Kozhedub Y.S., Plunien G., Brandau C., Stöhlker Th. // Phys. Rev. A. 2016. V. 93. P. 052502. doi 10.1103/PhysRevA.93.052502

[10] Zubova N.A., Anisimova I.S., Kaygorodov M.Yu., Kozhedub Yu.S., Malyshev A.V., Shabaev V.M., Tupitsyn I.I., Kozhedub Y.S., Plunien G., Brandau C., Stöhlker Th. // J. Phys. B. 2019. V. 52. P. 185001. doi 10.1088/13616455/ab3148

[11] Artemyev A.N., Shabaev V.M., Yerokhin V.A., Plunien G., Soff G. // Phys. Rev. A. 2005. V. 71. P. 062104. doi 10.1103/PhysRevA.71.062104

[12] Malyshev A.V., Kozhedub Y.S., Glazov D.A., Tupitsyn I.I., Shabaev V.M. // Phys. Rev. A. 2019. V. 99. P. 010501(R). doi 10.1103/PhysRevA.99.010501
[13] Kozhedub Y.S., Malyshev A.V., Glazov D.A., Shabaev V.M., Tupitsyn I.I. // Phys. Rev. A. 2019. V. 100. P. 062506. doi 10.1103/PhysRevA.100.062506

[14] Tupitsyn I.I., Shabaev V.M., Crespo Lopez-Urrutia J.R., Draganic I., Soria Orts R., Ullich J. // Phys. Rev. A. 2003. V. 68. P. 022511. doi 10.1103/PhysRevA.68.022511

[15] Tupitsyn I.I., Zubova N.A., Shabaev V.M., Plunien G., Stöhlker Th. // Phys. Rev. A. 2018. V. 98. P. 022517. doi 10.1103/PhysRevA.98.022517

[16] Shabaev V.M. // J. Phys. B. 1993. V. 26. P. 1103. doi 10.1088/0953-4075/26/6/011

[17] Papoulia A., Carlsson B. G., Ekman J. // Phys. Rev. A. 2016. V. 94. P. 042502. doi 10.1103/PhysRevA.94.042502

[18] Ekman J., Jönsson P., Godefroid M., Naze C., Gaigalas G., Bieron J. // Comput. Phys. Commun. 2019. V. 235. P. 433. doi 10.1016/j.cpc.2018.08.017

[19] Milstein A.I., Sushkov O.P., Terekhov I.S. // Phys. Rev. A. 2004. V. 69. P. 022114. doi 10.1103/PhysRevA.69.022114

[20] Yerokhin V.A. // Phys. Rev. A. 2011. V. 83. P. 012507. doi 10.1103/PhysRevA.83.012507

[21] Шабаев В.М. // Теор. мат. физ. 1985. V. 63. Р. 394; Shabaev V.M. // Theor. Math. Phys. 1985. V. 63. P. 588. doi

[22] Шабаев В.М. // Ядерная физика. 1988. V. 47. Р. 107 Shabaev V.M. // Sov. J. Nucl. Phys. 1988. V. 47. P. 69]. doi

[23] Pachucki K., Grotch H. // Phys. Rev. A. 1995. V. 51. P. 1854. doi 10.1103/PhysRevA.51.1854

[24] Artemyev A.N., Shabaev V.M., Yerokhin V.A. // Phys. Rev. A. 1995. V. 52. P. 1884. doi 10.1103/PhysRevA.52.1884

[25] Shabaev V.M. // Phys. Rev. A. 1998. V. 57. P. 59. doi 10.1103/PhysRevA.57.59

[26] Adkins G.S., Morrison S., Sapirstein J. // Phys. Rev. A. 2007. V. 76. P. 042508. doi 10.1103/PhysRevA.76.042508

[27] Malyshev A.V., Popov R.V., Shabaev V.M., Zubova N.A. // J. Phys. B: At. Mol. Opt. Phys. 2018. V. 51. P. 085001. doi 10.1088/1361-6455/aab29f

[28] Malyshev A.V., Anisimova I.S., Mironova D.V., Shabaev V.M., Plunien G. // Phys. Rev. A. 2019. V. 100. P. 012510. doi 10.1103/PhysRevA.100.012510

[29] Palmer C.W.P. // J. Phys. B: At. Mol. Phys. 1987. V. 20. P. 5987. doi 10.1088/0022-3700/20/22/011

[30] Kozhedub Y.S., Volotka A.V., Artemyev A.N., Glazov D.A., Plunien G., Shabaev V.M., Tupitsyn I.I., Stöhlker Th. // Phys. Rev. A. 2010. V. 81. P. 042513. doi 10.1103/PhysRevA.81.042513

[31] Naze C., Verdebout S., Rynkun P., Gaigalas G., Godefroid M., Jönsson P. // At. Data Nucl. Data Tables. 2014. V. 100. P. 1197. doi 10.1016/j.adt.2014.02.004

[32] Fischer C.F., Godefroid M., Brage T., Jönsson $P$, Gaigalas G. // J. Phys. B: At. Mol. Opt. Phys. 2016. V. 49. P. 182004. doi 10.1088/0953-4075/49/18/182004

[33] Filippin L., Bieron J., Gaigalas G., Godefroid M., Jönsson P. // Phys. Rev. A. 2017. V. 96. P. 042502. doi 10.1103/PhysRevA.96.042502

[34] Plunien G., Müller B., Greiner W., Soff G. // Phys. Rev. A. 1991. V. 43. P. 5853. doi 10.1103/PhysRevA.43.5853

[35] Plunien G., Soff G. // Phys. Rev. A. 1995. V. 51. P. 1119; Phys. Rev. A. 1996. V. 53. P. 4614. doi 10.1103/PhysRevA.53.4614.2

[36] Nefiodov A.V., Labzowsky L.N., Plunien G., Soff G. // Phys. Lett. A. 1996. V. 222. P. 227. doi 10.1016/03759601(96)00650-0

[37] Volotka A.V., Plunien G. // Phys. Rev. Lett. 2014. V. 113. P 023002. doi 10.1103/PhysRevLett.113.023002 
[38] Kozhedub Y.S., Andreev O.V., Shabaev V.M., Tupitsyn I.I., Brandau C., Kozhuharov C., Plunien G., Stöhlker T. // Phys. Rev. A. 2008. V. 77. P. 032501.

doi 10.1103/PhysRevA.77.032501

[39] Bemis Jr. C.E., McGowan F.K., Ford J.L.C., Milner W.T., Stelson P.N., Robinson R.L. // Phys. Rev. C. 1973. V. 8. P. 1466. doi 10.1103/PhysRevC.8.1466

[40] Zumbro J.D., Shera E.B., Tanaka Y., Bemis C.E., Naumann R.A., Hoehn M.V., Reuter W., Steffen R.M. // Phys. Rev. Lett. V. 53. P. 1888. doi 10.1103/PhysRevLett.53.1888

[41] Moller P., Nix J.R., Myers W.D., Swiatecki W.J. // At. Data Nucl. Data Tables. 1995. V. 59. P. 185. doi 10.1006/adnd.1995.1002

[42] Angeli I., Marinova K.P. // At. Data Nucl. Data Tables. 2013. V. 99. P. 69. doi 10.1016/j.adt.2011.12.006 